SCIENTIFIC REPORT

\title{
The use of low dose methotrexate in children with chronic anterior and intermediate uveitis
}

\section{A R Malik, C Pavesio}

Br J Ophthalmol 2005;89:806-808. doi: 10.1136/bjo.2004.054239

Aim: To assess the efficacy of low dose methotrexate (MTX) therapy for children with chronic anterior and intermediate uveitis.

Methods: A retrospective case review of 10 children who received MTX for chronic uveitis at a tertiary referral centre was performed. The following data were recorded for each patient: age, sex, race, duration of uveitis, primary diagnosis, anatomical localisation of uveitis, corticosteroid therapy, dose range of MTX, duration of MTX therapy, and side effects of MTX therapy. Several clinical parameters were evaluated to study the effect of MTX. These included visual acuity, anterior chamber inflammation, and topical and oral corticosteroid requirement.

Results: After MTX VA of $6 / 6$ or better was present in $100 \%$ right eyes and $80 \%$ left eyes $(p=0.055$ and $p=0.016$, respectively). Anterior chamber inflammation decreased in $60 \%$ of children after MTX $(p=0.0168)$. The requirement of topical steroid decreased from a mean of 5.6 times a day before MTX to 1.5 times a day after MTX $(p=0.005)$. The dose of oral steroid decreased from a mean of $18 \mathrm{mg}$ per day to $2.85 \mathrm{mg}$ per day $(p=0.012)$. The most common adverse effect was nausea (20\%). No patient required discontinuation of MTX because of side effects.

Conclusion: MTX is effective and safe for chronic anterior and intermediate uveitis in children. An increase awareness of its efficacy is required among paediatricians and ophthalmologists to prevent sight threatening complication of chronic uveitis and its treatment with long term use of steroids.

A lthough uveitis is relatively uncommon in children it is a serious, potentially blinding disease with large potential social and economic consequences. ${ }^{1}$ Juvenile idiopathic arthritis (JIA) is the most common cause followed by idiopathic uveitis and pars planitis. ${ }^{12}$ Corticosteroids are the mainstay of treatment but long term treatment in all routes of administration is associated with adverse effects with ocular and systemic manifestations. ${ }^{3-5}$

Because of the adverse effects associated with persistent uveitis or chronic use of corticosteroids it is recommended, for those in need of long term anti-inflammatory medication, to start corticosteroids in the acute stage of the disease and taper off subsequently with combined use of a steroid sparing medication. ${ }^{67}$ MTX is a folate analogue that inhibits dihydrofolate reductase, inhibiting the production of tetrahydrofolate, which is necessary for DNA synthesis. The immunosuppressive effects of low dose MTX are primarily through its antiproliferative effect on rapidly dividing immune cells. The secondary effects of MTX include cytopenia, hepatotoxicity, and interstitial pneumonitis.
A few well documented reports exist in the paediatric literature on the use of MTX in JIA associated uveitis. ${ }^{8}{ }^{9}$

In this retrospective case series we present our experience with the use of low dose MTX in 10 children with chronic low grade anterior and intermediate uveitis, including cases secondary to sarcoidosis, and idiopathic type.

\section{METHODS}

The clinical records of all children with uveitis, seen at a uveitis service at Moorfields Eye Hospital between 1999 and 2003 and who were treated with MTX, were reviewed. Initial examination of each patient included a complete medical and ophthalmic history and an eye examination. The number of cells in the anterior chamber was quantified by a standard grading scale $(0$, occasional, $1+$ to $4+)$ modified from Hogan et al. ${ }^{10}$ Before initiation of MTX a complete blood count, liver function, and renal function tests were performed. These were monitored periodically for drug toxicity every 4 weeks.

The follow up of these patients before and during treatment was in close liaison with a paediatrician, who also carried out investigations for the diagnosis of any systemic associated disease.

The following data were recorded for each patient: age, sex, race, duration of uveitis, primary diagnosis, anatomical localisation of uveitis, corticosteroid therapy, dose range of MTX, duration of MTX therapy, and side effects of MTX therapy.

Several clinical parameters were evaluated to study the effect of MTX. These included visual acuity, anterior chamber inflammation, and topical and oral corticosteroid requirement.

\section{Statistical analysis}

Data from the last clinic visit before initiating MTX were compared with those from the last available visit while on MTX by using the Wilcoxon signed rank test. The $\mathrm{p}$ values of 0.05 or less were considered statistically significant.

\section{RESULTS}

Ten children (all had age of onset of uveitis at less than 16 years of age) who had treatment with MTX were identified and the data were analysed. Table 1 lists the patients, sex, dose and duration of MTX, initial and final topical and oral steroid doses, and initial and final visual acuities. There were eight girls and two boys. The mean age was 11.9 years (range $7-16$ years). Three patients were white, three were Asian Indians, two were Afghani, one was Chinese, and one was African. The paediatrician in charge of the case was responsible for all investigations to exclude an underlying disease, including serology, ANA, SACE, lysozyme, imaging, or any tests considered necessary. The diagnosis of uveitis was idiopathic in seven cases and in three cases the diagnosis of presumed sarcoidosis was

Abbreviations: JIA, juvenile idiopathic arthritis; MTX, methotrexate 


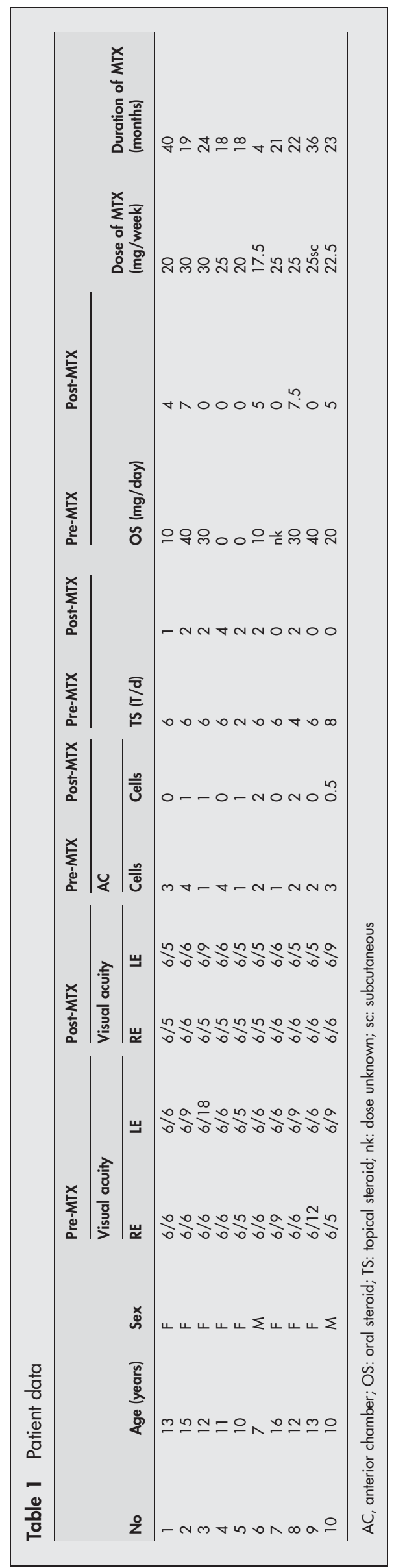

established on clinical grounds by the paediatrician. The location of uveitis was anterior in six and intermediate in four cases. The uveitis was bilateral in all the children.

The mean duration of uveitis before commencement of MTX was 24 months (range 6-36 months).

The indication for initiating MTX in all these children was either poor control of inflammation or development of side effects from conventional therapy with corticosteroids. Patients 3 and 4 (table 1) had also received ciclosporin before introduction of MTX, and patient 4 continued with a combination of both drugs. Introduction of MTX was only considered after previous therapy was thought to be a failure, following a period of 2 months of use. All children were treated with oral MTX except one (table 1, child no 9) who was unable to tolerate oral MTX because of severe nausea and remained on subcutaneous MTX (sc) throughout the study. All children were treated with low dose once weekly oral or sc MTX for a mean of 22.5 months (range 4-40 months) at the time of analysis. All children received folic acid $2.5-5 \mathrm{mg} /$ day from the start of MTX therapy.

Nausea was the only common complication associated with MTX use. One child receiving combination therapy (MTX and ciclosporin), reported abdominal pain, but no change in therapy was necessary. Only one patient (case 9) had a small rise of ALT of $47(0-40)$. No other patient had abnormality of liver function tests. No abnormalities in blood count or renal function were detected in any of the children.

Ocular complications associated with steroid use were ocular hypertension in two patients, which resolved following better control of inflammatory activity with MTX and reduction of topical steroid therapy, and cataract in two patients. One patient developed cystoid macular oedema during activity of the disease, which resolved following MTX therapy (right eye of patient 9).

Snellen visual acuity of $6 / 6$ or better was present in eight $(80 \%)$ right eyes and six $(60 \%)$ left eyes before MTX. After MTX therapy VA was $6 / 6$ or better in $10(100 \%)$ right eyes $(p=0.055)$ and in eight $(80 \%)$ left eyes $(p=0.016)$ (table 1$)$.

After MTX anterior chamber inflammation decreased in $60 \%$ (six children). In four children there was no change in inflammation. The decrease in inflammation after MTX was statistically significant $(p=0.0168)$ (table 1 ).

The topical corticosteroid frequency was six times per day in $70 \%$ of the children before MTX. After MTX, at the final visit, only one child was using topical steroid four times per day. The difference between pre-MTX and post-MTX topical steroid frequency was statistically significant $(p=0.005)$ (table 1).

Seven children were using oral steroids before MTX. The dose of oral prednisolone was decreased in all children after MTX. The mean initial dose was $18 \mathrm{mg}$ (range 10-40 mg) and the mean final dose was $2.85 \mathrm{mg}$ (range 5-7.5 mg). This difference between pre-MTX and post-MTX oral steroid use was statistically significant $(\mathrm{p}=0.012)$.

The most common adverse effect was nausea (patients 9 and 10); however, this was mild in intensity and well tolerated. No patient required discontinuation of MTX as a result of adverse effects.

\section{DISCUSSION}

Uveitis in children is a serious disease which may go unrecognised because it may be asymptomatic or because of its insidious nature. The child may already have serious complications of chronic uveitis at the time of presentation to the ophthalmologist. The ophthalmologist is therefore posed with the challenge of prompt control of inflammation and preventing further development of ocular and systemic complications. The current therapy of choice is corticosteroids, topical initially for the anterior forms and subsequently 
oral, when that fails, or in the presence of posterior disease (intermediate uveitis type). Long term systemic corticosteroids have an adverse effect on the developing skeletal and immune system.

Low dose, once a week MTX has become the therapeutic agent of choice for children with JIA who fail to respond adequately to the non-steroidal anti-inflammatory drugs. In these selected patients with chronic active uveitis low dose MTX was effective and well tolerated. At the time MTX was started all children had active uveitis despite frequent instillation of topical steroids and previous administration of oral steroids. After treatment with MTX there was significant reduction in the severity of uveitis.

Several authors have reported a favourable response of JIA associated uveitis to treatment with low dose MTX. In low doses MTX is less toxic than most immunosuppressants including corticosteroids.

Weiss et al describe the use of low dose subcutaneous MTX in seven children with JIA associated uveitis. All patients had chronic active uveitis for at least 1 year (range $1-17$ years) inadequately suppressed with corticosteroids and had progressive steroid related cataract and glaucoma. ${ }^{9}$ The use of MTX decreased the severity of uveitis in six of seven patients and allowed for the discontinuation or reduction of corticosteroid. In another case series low dose oral MTX therapy was found to be effective in the treatment of four children with severe iritis secondary to JIA and sarcoidosis. ${ }^{11}$

The use of systemic therapy in these patients differs from conventional indication for introduction of such agents. Most of the time, they are introduced in vision threatening uveitis when visual acuity falls below 6/12. In our patients the decision to introduce systemic therapy was based on the presence of severe anterior segment inflammation, in spite of frequent topical therapy. None of our patients had significant change in visual acuity as the justification for therapy.

Overall, MTX is well tolerated and despite its side effect profile, it continues to have a role as a corticosteroid sparing agent in the management of patients with ocular inflammatory disease. ${ }^{12}$ Informed opinion would indicate that it should be used early in disease to prevent permanent damage to intraocular structure.

\section{Authors' affiliations}

A R Malik, East Surrey Hospital, Redhill, Surrey, UK

C Pavesio, Moorfields Eye Hospital, London ECIV 2PD, UK

Correspondence to: C Pavesio, MD, FRCOphth, Moorfields Eye Hospital, London ECIV 2PD, UK; carlos.pavesio@moorfields.nhs.uk

Accepted for publication 12 November 2004

\section{REFERENCES}

1 Tugal-Tutkin I, Havrlikova K, Power WJ, et al. Changing patterns in uveitis in childhood. Ophthalmology 1996;103:375-83.

2 Kanski JJ, Shun-Shin A. Systemic uveitis syndromes in childhood. An analysis of 340 cases. Ophthalmology 1984:91247-51.

3 Solomon SD, Cunningham Jr ET. Use of corticosteroids and non-corticosteroid immunosuppressive agents in patients with uveitis. Comprehensive Ophthalmology Update 2001;1:273-86.

4 Nussenblatt RB, Whitcup SM, Palestine AG. Uveitis, fundamentals and clinical practice. 2nd ed. St Louis: Mosby, 1996:97-129

5 Jabs DA, Rosenbaum JT, Foster CS, et al. Guidelines for the use of immunosuppressive drugs in patients with ocular inflammatory disorders: recommendations of an expert panel. Am J Ophthalmol 2000;130:492-513.

6 Rothova A. Corticosteroids in uveitis. Ophthalmol Clin N Am 2002:389-94.

7 Tamesis RR, Rodriguez A, Christen GW, et al. Systemic drug toxicity trends in immunosuppressive therapy of Immune and Inflammatory ocular disease. Ophthalmology 1996;103:768-75.

8 Hemady R, Baer J, Foster C, et al. Immunosuppressive drugs in the management of progressive corticosteroid-resistant uveitis with juvenile rheumatoid arthritis. Int Ophthalmol Clin 1992;32:241-52.

9 Weiss HA, Wallace AC, Sherry DD. Methotrexate for resistant chronic uveitis in children with juvenile rheumatoid arthritis. J Pediatr 1998;133:266-8.

10 Hogan MJ, KimuraSJ, Thygeson P. signs and symptoms of uveitis. 1. Anterior uveitis. Am J Ophthalmol 1959;47:155-170.

11 Shetty KA, Zganjar EB, Ellis SG, et al. Low-dose methotrexate in the treatment of severe juvenile rheumatoid arthritis and sarcoid iritis. J Pediatr Ophthalmol Strabismus 1999:36:125-8.

12 Dialilian AR, Nussenblatt RB. Immunosuppression in uveitis. Ophthalmol Clin N Am 2002;15:395-404.

\section{Call for papers}

11 th European Forum on Quality Improvement in Health Care 26-28 April 2006, Prague, Czech Republic

Deadline 30 September 2005.

For further information and to submit online go to: www.quality.bmipg.com 